\title{
Das Hören des Cochlea-Implantats
}

\author{
von Beate Ochsner und Robert Stock
}

\section{Einleitung}

Das Cochlea-Implantat (CI) soll die Funktion eines nicht intakten Innenohres ersetzen und den Patienten durch direkte Reizung der funktionierenden Hörnervenfasern einen Höreindruck vermitteln: ,[Die] Ströme werden an die angekoppelten Hörnervenfasern weitergegeben und zum Gehirn zur weiteren Verarbeitung transportiert. Der Patient hört. " ${ }^{\text {DI }}$ Dieses hier als simples Prinzip dargestellte zu erreichende Resultat eines Hörens mit CI wird vor allem aus medizinischer Sicht immer wieder als Erfolgsgeschichte einer elektronischen Neuroprothese dargestellt, die im Wesentlichen auf Forschungen von Pionieren wie Hallowell Davis an der Harvard Medical School in den 1930er Jahren basiert und die seit den 1950er Jahren mit und durch die Arbeiten von französischen, australischen sowie US-amerikanischen Medizinern weitergeschrieben wurde. ${ }^{2}$ Noch in der Zeit der klinischen Erprobung wurde 1978 das erste Mehrkanal-CI der Medizingeschichte in Australien implantiert, in Deutschland führte Ernst Lehnhardt 1984 die erste CI-Implantation durch und nur vier Jahre später wurde das erste gehörlose Kind mit einem CI versorgt. ${ }^{3}$ Seit dieser Zeit nimmt die Zahl an Implantationen stetig zu. Einer Statistik der Deutschen Cochlea Implantat Gesellschaft e.V. zufolge gibt es weltweit 300000 CI-Träger, davon in Deutschland circa $30000 .^{4}$

Zahlreiche Autoren, die kultur- oder technikgeschichtliche Darstellungen des CI schreiben, setzen den Ausgangspunkt für das Narrativ apparativen Hörens bei elektrophysiologischen Versuchen an. ${ }^{5}$ Als Vorläufer des CI werden in der Regel die Ex-

1 Thomas Lenarz/Karl Friedrich Mack, Das Cochlea-Implantat aus medizinischer Sicht, in: Spektrum der Wissenschaft, 1.1.2000.

2 Vgl. u. a. Raylene Paludneviciene/Irene Leigh, Cochlear implants, Evolving perspectives. Washington 2011; John K. Niparko, Cochlear implants. Principles \& practices. Philadelphia 2009.

$3 \mathrm{Vgl}$. Anne Ernst/Rolf Dieter Battmer/Ingo Todt, Cochlear Implant heute, Heidelberg 2009.

4 Vgl. Statistik der Deutschen Cochlear Implant Gesellschaft e. V., Stand: 31. Dezember 2011, in: Schnecke Online, http://schnecke-online.de/informieren/behandlung-und-reha/cochlea-implantat. html (02.09.2014).

5 Vgl. u.a. William F. Agnew, Neural prostheses. Fundamental studies, Englewood Cliffs, NJ 1990, 7; Saurabh B. Shah/Jeannie H. Chung/Robert K. Jackler, Lodestones, Quackery, and Science. Electrical Stimulation of the Ear Before Cochlear Implants, in: Otology \& Neurotology 18 (1997) H. 5, 665-670, 665; Andras Gedeon, Science and technology in medicine. An illustrated account based on ninety-nine landmark publications for five centuries, New York, NY 2006, 145; J. Thomas Roland Jr. /Tina C. Huang/Andrew J. Fishman, Cochlear Implant Electrode History. Choices, and Insertion Techniques, in: Susan B. Waltzman/J. Thomas Roland (Hg.), Cochlear implants, New York 2006, 110-125, 110. 
perimente Alessandro Voltas angeführt, der um 1800 in Versuchsanordnungen mit elektrischem Hören experimentierte. So wird berichtet, der Physiker habe sich die Gehörgänge mit Kochsalzlösung gefüllt und über zwei Elektroden aus seiner selbstentwickelten Batterie eine Spannung angelegt. ${ }^{6}$ Diese Konstellation gilt als wegweisend für die elektrische Reizung des noch funktionsfähigen Hörnervs mittels intracochleärer Stimulationselektroden. Auf der einen Seite wird die Geschichte des CI auf diese Weise in die experimentelle Begründung der Elektrophysiologie durch Galvani bzw. ihre Weiterentwicklung durch Volta Ende des 18. und Anfang des 19. Jahrhunderts eingeschrieben. Auf der anderen Seite existiert ein weiterer Diskurs, der die Medikalisierbarkeit eines zwar nicht (mehr) vollständig funktionstüchtigen, wohl aber noch leitungsfähigen Körpers durch künstliche elektrische Reizung zum Gegenstand hat. So entsteht das Konzept eines signalverarbeitenden und -prozessierenden Körpers, der in seiner Eigenschaft als Leitungsmedium selbstregulierende Rückkopplungsprozesse durchführen und auf diese Weise ,funktional passend gemacht ${ }^{\text {“7 }}$ werden kann. Damit wird eine zukunftsträchtige und Erfolg verheißende Schnittstelle zwischen Elektrophysiologie, Kybernetik und Medizin bzw. Prothetik eröffnet. Der folgende Artikel möchte einen Beitrag zur Fort- bzw. Umschreibung dieses Narrativs leisten und untersucht die (Selbst-)Beschreibungen von Hörerlebnissen durch CI-Träger_innen im Rahmen ihrer Aktivierungs- und audiologischen Einstellungsphasen. ${ }^{8}$ Diese automedialen Zeugnisse ${ }^{9}$ werden als Elemente eines Individuationsprozesses begriffen, im Rahmen dessen ,die CI-Träger_in', das CI selbst und verschiedenen Formen von Vergemeinschaftungen (CI-Gemeinschaft, Patientenkollektive, Selbsthilfegruppen, Elterninitiativen, Deutsche Cochlea Gesellschaft e. V., Cochlea-Zentren verschiedener Universitätsklinika, etc.) wechselseitig verfertigt werden. ${ }^{10}$ Die zentralen Phasen

6 Volta leitete den mit seiner Batterie produzierten Strom über zwei Metalldrähte in seine Ohren: „At the moment when the circle was thus complete I received a shock in the head, and some moments after I began to hear a sound, or rather noise in the ears, which I cannot well define; it was kind of a crackling with shocks, as if some paste or tenacious matter had been boiling. This noise continued incessantly, and without increasing, all the time that the circle was complete. The disagreeable sensation, which I apprehended might be dangerous, of shock in the brain, prevented me from repeating the experiment." Zitiert nach Shah/Chung/Jackler, Lodestones, 666.

7 Karin Harrasser, Sensible Prothesen. Medien der Wiederherstellung von Produktivität, in: Body Politics 1 (2013), H. 1, 99-117, 103. An anderer Stelle spricht Harrasser auch vom Konzept einer kybernetischen Anthropologie, die - da kybernetisch - keine mehr ist, gleichwohl Lebewesen beschreibt, die ihren Umweltbezug als signalverarbeitende Entität regulieren und auf diese Weise immer wieder versuchen, ins Gleichgewicht zu kommen. Vgl. Karin Harrasser, Passung durch Rückkopplung. Konzepte der Selbstregulierung in der Prothetik des Ersten Weltkriegs, in: Stefan Fischer/Erik Maehle/Rüdiger Reischuk (Hg.), Informatik 2009. Im Focus das Leben, Bonn 2009, 788-801.

$8 \mathrm{Vgl}$. Madeleine Akrich, Vom Objekt zur Interaktion und zurück. Eine Diskussion mit Madeleine Akrich, Antoine Hennion und Vololona Rabeharisoa. Moderiert durch Lorenza Mondada, in: Zeitschrift für qualitative Bildungs-, Beratungs- und Sozialforschung 5 (2004) H. 2, 239-271.

9 Der von Dünne/Moser geprägte Begriff der „Automedialität“ bezeichnet die produktive Verschränkung der Autobiographieforschung mit der Medienwissenschaft. Das Konzept erweitert ältere Ansätze nicht nur um die allgemeine Relevanz der Medialität von Selbstdarstellungen, sondern zeigt die Wechselbeziehungen zwischen Subjektkonstitution und Wissensgeschichte auf. Vgl. Jörg Dünne/Christian Moser, Allgemeine Einleitung. Automedialität, in: dies. (Hg.), Automedialität. Subjektkonstitution in Schrift, Bild und neuen Medien, München 2008, 7-16.

10 Es wird von einem relationalen Verhältnis zwischen Formen von Subjektivierungen und Ver- 
der Aktivierung des CI sowie der reziproken Einstellung von Gerät und Träger_in werden dabei von Akteur zu Akteur durchaus unterschiedlich betrachtet und gewichtet: Während es Mediziningenieuren oder Audiologen vornehmlich darum geht, die Interaktionen zwischen CI und Usern durch verschiedene technische und organisatorische Entscheidungen zu stabilisieren und mithin das soziotechnische Gefüge zu sichern, ${ }^{11}$ stehen bei anderen ausschließlich die ,realen " Handlungen innerhalb eines bestimmten Interaktionsrahmens im Vordergrund, ohne dessen Entwicklung oder Bedingungen zu reflektieren. ${ }^{12}$ Aus medienwissenschaftlicher Sicht sind nun vorrangig diejenigen soziomedialen Operationen von Interesse, die solche Art von Rahmungen ermöglichen und zumindest temporär stabilisieren. ${ }^{13}$ So werden im Folgenden diejenigen akustischen Effekte analysiert, die durch das CI, seine Erstaktivierung und die späteren Einstellungen hervorgebracht werden. Deren subjektive Wahrnehmung wird zugleich durch die in die Schaltkreise eingebundenen Personen in den Mittelpunkt gerückt. Dabei geht es darum herauszuarbeiten, wie sich die Produktion einer kulturellen Praxis des CI-Hörens im Rahmen eines bestimmten soziotechnischen Arrangements vollzieht.

In dieser Perspektive werden die Aktivierungs- und Einstellungsprozesse als Experimentalräume (und -zeiten) begriffen, innerhalb derer ein spezifisches Wissen über CI-Hörende hergestellt wird und die das CI als epistemisches Objekt formatieren. ${ }^{14}$ Dieses Wissen wird durch Selbstbeschreibungen in Form von transepistemischen ${ }^{15}$,

gemeinschaftungen ausgegangen, die einen nicht abgeschlossenen Prozess bezeichnen und sich im Kontext eines sich wandelnden soziotechnischen Gefüges (hier der CI-Handlungszusammenhang) situieren. Zum Individuationsprozess vgl. Gilbert Simondon, L'individuation à la lumière des notions de forme et d'information, Grenoble 2005; ders., L'individuation psychique et collective, Paris 2007 ; ders., Ergänzende Anmerkungen zu den Konsequenzen des Individuationsbegriffes, in: Ilka Becker/Michael Cuntz/Astrid Kusser (Hg.), Unmenge. Wie verteilt sich Handlungsmacht?, München 2008, 47-74. Siehe auch Michel Callon, Some elements of a sociology of translation: domestication of the scallops and the fishermen of St Brieuc Bay, in: John Law (Hg.), Power, action and belief: a new sociology of knowledge?, London 1986, 196-223.

11 Vgl. Sebastian Hoth, Audiometrie - Die Untersuchung des Gehörs und seine technische Versorgung, in: Rüdiger Kramme (Hg.), Medizintechnik, Berlin u. a. 2011, 217-270, 265-270; Marc D. Eisen, Djourno, Eyries, and the First Implanted Electrical Neural Stimulator to Restore Hearing, in: Otology \& Neurotology 24 (2003) H. 3, 500-506, 503.

12 So beispielsweise bei den Usern Goffmans: Vgl. Erving Goffman, Interaktionsrituale über Verhalten in direkter Kommunikation, Frankfurt a. M. 1971.

13 Latour geht davon aus, dass eine Untersuchung von Beziehungsverhältnissen zwischen Menschen, Praktiken und Objekten eingegrenzt werden muss, damit sie nicht ko-extensiv werden. Dies bedeutet, dass ein Akteur nur durch die ,,isolierende Wirkung eines Rahmens, der zudem den Rest seiner Geschichte und die seiner anderen Partner außerhalb des Geschehens lässt" mit einem anderen Akteur interagieren kann: „Es gilt, gerade diese Suspendierung, diese partielle Unterbrechung, diese Klausur zu verstehen, innerhalb derer sich die Interaktion entfalten kann, ohne sich mit allen anderen zu überschneiden. " Bruno Latour, Eine Soziologie ohne Objekt? Anmerkungen zur Interobjektivität, in: Berliner Journal für Soziologie 11 (2001) H. 2, 237-252, 239.

14 Vgl. Hans-Jörg Rheinberger, Sichtbar Machen. Visualisierung in den Naturwissenschaften, in: Klaus Sachs-Hombach (Hg.), Bildtheorien. Anthropologische und kulturelle Grundlagen des Visualistic Turn, Frankfurt a. M. 2009, 127-146, 138.

15 Vgl. Karin Knorr-Cetina, Scientific Communities or Transepistemic Arenas of Research? A Critique of Quasi-Economic Models of Science, in: Social Studies of Science 12 (1982), 101-130; dies., ,Viskurse“ der Physik. Wie visuelle Darstellungen ein Wissenschaftsgebiet ordnen, in: Gottfried Boehm/Jörg Huber (Hg.), Konstruktionen Sichtbarkeiten: Interventionen, Wien - New York 1999, 245-263. 
d.h. kontextuell und medial mitbestimmten Praktiken, in diesem Fall Blogs, sichtund lesbar gemacht. Dabei führt das ,Erschreiben ‘ bestimmter Formen des CI-Hörens durch die Blogger_innen dazu, die im klinischen Zusammenhang etablierten Interaktionsrahmungen zu problematisieren, zu verunsichern, aber möglicherweise auch zu bestätigen. Deutlich wird aber auch, dass beim CI-Hören ähnliche Konfigurationen wie beim sog. ,normalen ' Hören oder Nicht-Hören zu beobachten sind, da es sich in allen Fällen nicht um biologisch-essentialistische Eigenschaften, sondern um Produkte kultureller Praktiken handelt. So wird im Folgenden darauf geachtet, Dichotomien zwischen einer sog. hörenden bzw. nicht-hörenden „Welt“ nicht zu reproduzieren, sondern vielmehr die graduellen Unterschiede in den Praktiken wie auch ihrer Diskursivierung zu beschreiben.

Durch die massive Verbreitung des CI seit den 1990er Jahren existiert mittlerweile eine Fülle an Erfahrungsberichten und Memoiren, in denen CI-Träger_innen über ihren Umgang mit der implantierten Technologie berichten. Solche lebensgeschichtlichen Texte finden sich vermehrt in Internet-Blogs, die in Form und Aufbau dem klassischen Tagebuch ähneln, wobei die digitale Bedingung nicht nur die Integration unbewegter, sondern auch bewegter Bilder erlaubt. In paradigmatischer Weise steht die US-Amerikanerin Sarah Churman für diese Form der Subjektivierungspraxis, wenngleich an dieser Stelle spezifiziert werden muss, dass Churman kein CI, sondern ein Mittelohrimplantat mit dem Namen Esteem der Firma Envoy Medical trägt. ${ }^{16}$ Die Video-Aufnahme ihrer Erstaktivierung wurde als Youtube-Video millionenfach abgerufen, ihre Erfahrungen und Erlebnisse können in ihrem Blog gelesen werden, und vor kurzem stellte sie ihr Buch Powered on vor. ${ }^{17}$ Dieser sensationellen Erfolgsgeschichte stehen Kommentare wie derjenige William Magers gegenüber, der ganz andere Erfahrungen mitteilt: ,The switch on is usually the worst day of most people's lives. “18 Weiterhin werden im Folgenden die Selbstbeschreibungen von Michael Chorost, ${ }^{19}$ Alexander Görsdorf, ${ }^{20}$ Enno Park ${ }^{21}$ oder auch Peggy Rußat ${ }^{22}$ zur Analyse des CI-Hörens

16 Zweifel bezüglich der Vorstellung, dass Churman prälingual ertaubte, äußert der Envoy-Manager Patrick Spearman. Vgl. David Kirkwood, Envoy CEO discusses the world's most famous Esteem device patient, in: Hearing News Watch, 18.11.2011, http://hearinghealthmatters.org/ hearingnewswatch/2011/envoy-ceo-discusses-the-world's-most-famous-esteem-device-patient/ (01. 10.2014)

17 Vgl. Sarah Churman, Powered on. The SOUNDS I Choose to Hear and the NOISE I Don't, Pensacola, FL 2012. Für den Blog vgl. Sarah Churman, In the world, but not of the world, http:// sarahchurman.blogspot.de/ (26.06.2014). Das Video der Erstaktivierung: 29 years old and hearing myself for the 1st time, in: youtube, http://www.youtube.com/watch?v=LsOo3jzkhYA (15. 07. 2014).

18 Zitiert nach Switching on my hearing - William's cochlear implant story. BBC Ouch! It's a disability thing, http://www.bbc.co.uk/blogs/legacy/ouch/2013/01/switching_on_my_hearing_-_will. html (17.06.2014).

19 Vgl. Michael Chorost, Blog, http://michaelchorost.com/blog/ (26.06. 2014). Siehe auch Michael Chorost, Rebuilt. How becoming part computer made me more human, London 2006.

20 Görsdorf führt den Blog Not quite like Beethoven. Ein Blog über Unhörbares, Unerhörtes, und Nichtgehörtes, http://notquitelikebeethoven.wordpress.com (16.06.2014). Eine Auswahl der Einträge des Blogs wurde kürzlich in Buchform veröffentlicht: Alexander Görsdorf, Taube Nuss. Nichtgehörtes aus dem Leben eines Schwerhörigen, Reinbek bei Hamburg 2013.

21 Vgl. Enno Park, die ennomane, http://www.ennomane.de/ (26.06.2014).

22 Vgl. Peggy Rußat, Mein Leben mit dem Cochlea Implantat, http://cochlearimplanttraegerin. de.tl/ (26.06.2014). 
als kulturelle Subjektivierungspraxis herangezogen, die, wie im Falle Görsdorfs oder auch Chorosts, häufig zuerst auf Blogs erschienen und später in Buchform publiziert wurden. Aus Gründen der Genauigkeit muss angeführt werden, dass es sich hierbei um sogenannte postlingual oder spätertaubte Personen handelt, ${ }^{23}$ die aufgrund der vor der Ertaubung gesammelten Hör- und Sprecherfahrungen eine besondere Position im Handlungszusammenhang CI einnehmen. ${ }^{24}$

\section{Blogs, Memoiren und CI-Hören}

In seinem Buch Signifying bodies. Disability in contemporary life writing unterscheidet G. Thomas Couser zwischen „,somebody memoir" und „nobody memoir“.25 Während bekannte Persönlichkeiten a priori als „,somebody“ gelten, müssten in der Öffentlichkeit nicht bekannte Personen sich erst einen Adressatenkreis schaffen. Couser arbeitet in seiner Studie heraus, dass das Genre des life writing eine bevorzugte Form der ,,nobody memoir“ darstellt. Es biete nicht nur Raum für die Reflexion der eigenen Lebensgeschichte, sondern auch für den Umgang und das Leben mit verschiedensten Behinderungen und den damit verknüpften körperlichen Befindlichkeiten. Gerade in Bezug auf autobiografische Darstellungen gehörloser Menschen ergeben sich dabei Spannungen: Diese resultieren einerseits aus den - teils geringen - Lese- und Schreibkompetenzen Gehörloser, denen andererseits ein wachsendes Interesse seitens der Deaf Community an Geschichten derer gegenübersteht, die sich ihr zugehörig fühlen, da die schriftlichen Zeugnisse eine eigene Deaf-CommunityGeschichte hervorbringen. ${ }^{26}$ Dabei vermitteln diese Werke häufig Innenansichten, welche die Perspektiven der Mehrheitsgesellschaft kritisch diskutieren oder stereotypen Auffassungen von Gehörlosigkeit widersprechen.

Dies betrifft in besonderer Weise die Praxis des Bloggens. ${ }^{27}$ Ohne thematische Vorgaben kann nahezu alles Anlass für Blogger darstellen, sich zu äußern, diesen spezifischen Text zu posten und dann von den Lesenden kommentieren zu lassen. Dabei handelt es sich um Formate, die auf der Basis der durch den Blog vermittelten Möglicgkeiten zur Selbstdarstellung einen „sich medial im Schreiben konstituierenden

23 Vgl. Anja Kittlitz, Andershörend. Die lebensweltliche Konstruktion des Schwerhörigseins, ein Beitrag aus kulturwissenschaftlicher Sicht, München 2012, $12 \mathrm{f}$.

24 Weitere Untersuchungen, die neben den genannten Medien auch Amateurvideos, Dokumentarfilme und Fernsehmagazinbeiträge berücksichtigen und das Thema also auf einer breiteren Materialbasis diskutieren, sind im Rahmen eines größeren Forschungsprojekts geplant.

$25 \mathrm{Vgl}$. G. Thomas Couser, Signifying bodies. Disability in contemporary life writing, Ann Arbor 2009, 1-4.

26 Vgl. Brenda Jo Brueggemann, Narrating Deaf Lives. Placing Deaf Autobiography, Biography and Documentary, in: dies., Deaf subjects. Between identities and places, New York u.a. 2009, 72-97; dies., ,Writing Insight‘: Deafness and Autobiography, in: American Quarterly 52 (2000) H. 2, 316-321; G. Thomas Couser, Signs of Life. Deafness and Personal Narrative, in: ders., Recovering bodies. Illness, disability, and life-writing, Madison, WI u. a. 1997, 221-287.

27 Vgl. Greg Myers, Discourse of blogs and wikis, London u.a. 2010; Axel Bruns, Blogs, Wikipedia, Second Life, and beyond. From production to produsage, New York u. a. 2008; Ina Ragnhild Langenfeld, Die Welt bloggt. Tagebuch und Weblogs im Vergleich, Marburg 2008; Jill Walker Rettberg, Blogging, Cambridge u. a. 2008. 
Selbstbezug“ ${ }^{\text {228 }}$ erst herstellen. Was beschrieben wird, geht zurück auf die Reflexion und das Sich-in-Beziehung-Setzen zu sich selbst im Moment des Schreibens. Im Fall der CI-Blogger re-produziert dies das mittels des technischen Objekts ermöglichte Sich-Selbst-Hören. Somit sind Blog-Einträge als situative Artikulationen zu verstehen, in denen unter bestimmten Bedingungen die auditiven Erlebnisse und Erfahrungen der Blogger re-konstruiert und in die Schriftsprache übersetzt werden. Die Praxis, auf diese Weise Auskunft über sich selbst und die Beziehung zu sich selbst zu geben, ähnelt anderen Selbstpraktiken wie z. B. der Beichte und daraus hervorgehenden säkularen Bekenntnistechniken wie dem Talkshow-Gespräch, Daily Soaps oder die bereits erwähnten Genres der Memoiren und des Tagebuchs. ${ }^{29}$ Gemeinsam ist allen diesen unterschiedlichen Formen, dass die Aussage über sich selbst in einem bestimmten Medium vollzogen wird und dass damit ein spezifischer Subjektivierungsprozess - nicht aber die abgeschlossene Produktion eines Subjekts - einhergeht. Ein solches ,Herbeischreiben ' des Selbst verlangt darüber hinaus eine gewisse Kontinuität. Bloggen ist folglich mit der Zumutung des regelmäßigen Schreibens (über sich selbst) verbunden, die Struktur des Blogs ist darauf angelegt, beständig neue Einträge aufzunehmen und diese über Newsfeeds zugänglich zu machen.

Im Falle der CI-Blogger ist das Schreiben mit einer Reflexion darüber verbunden, wie sich das Hören mit dem Implantat zu unterschiedlichen Zeiten und in unterschiedlichen Situationen darstellt und welche Maßnahmen von den Schreibenden ergriffen werden, um Veränderungen ihrer auditiven Wahrnehmung in Richtung einer Normalisierung herbeizuführen. Es zeigt sich dabei, dass das für das Schreiben des Blogs relevante Regime in abgewandelter Form auch als Selbsttechnik für das CI-Hören Bedeutung hat. Was CI-Blogger in ihren Einträgen schildern, sind nicht nur die von ihnen an sich selbst beobachteten Höreindrücke. Vielmehr wird auch die „Arbeit an sich selbst" bzw. die Normalisierungspraxis in einem neoliberalen Sinne erkennbar, die auf eine Verschaltung von Arbeitswissenschaft, Technologie und Medizin im Kontext einer Wissensgeschichte der Prothetik verweist. ${ }^{30}$ Es geht darum, Fortschritte im Bereich des Hörens kenntlich zu machen oder Rückschritte zu plausibilisieren. Vor allem steht aber die kontinuierliche Auseinandersetzung mit der Situation des CI-Hörens im Mittelpunkt. Vor diesem Hintergrund erscheint es dann auch als folgerichtig, dass die Blogs einen Ort bieten, an dem hauptsächlich Erfolgsgeschichten erzählt werden. Erfahrungen des Scheiterns sind anhand des vorgefundenen Materials nur selten zu

28 Dünne/Moser, Automedialität, 11.

$29 \mathrm{Vgl}$. Beate Ochsner, Image 2.0 oder: Selbsttechnologien in sozio-medialen Netzwerken, in: Vincent Kaufmann/Ulrich Schmid/Dieter Thomä (Hg.), Das öffentliche Ich. Selbstdarstellungen im literarischen und medialen Kontext, Bielefeld 2014, 161-178; Hannelore Bublitz, Im Beichtstuhl der Medien. Die Produktion des Selbst im öffentlichen Bekenntnis, Bielefeld 2010; Berthold Unfried, „Ich bekenne.“ Katholische Beichte und sowjetische Selbstkritik, Frankfurt a. M.u.a. 2006, 144-148; Hubert L. Dreyfus/Rabinow Paul, Michel Foucault jenseits von Strukturalismus und Hermeneutik, Frankfurt a. M. 1987, 205-210.

30 Dabei geht es vor allem seit dem Ersten Weltkrieg auch immer um die konkrete Wiederherstellung versehrter Menschen für den Arbeitsmarkt bzw. um die - wie in der audiologischen Einstellung - ständige Verbesserung des Hörens bzw. des Selbst. Vgl. Harrasser, Passung durch Rückkopplung. Siehe auch Ulrich Bröckling, Das unternehmerische Selbst. Soziologie einer Subjektivierungsform, Frankfurt a. M. 2007. 
beobachten, obwohl einzelne Rückschritte bzw. Probleme im Umgang mit dem Implantat sehr wohl zur Sprache gebracht werden. Im Folgenden soll aufgezeigt werden, dass die (Selbst-)Reflexion auf das CI-Hören ein dreiphasiges Normalisierungsmodell etabliert, das auf die Erstaktivierung eine Zeit der Einstellung und der disziplinierenden Übung folgen lässt. ${ }^{31}$

\section{Das Hören des Cochlea-Implantats}

\subsection{Erstaktivierung}

Die Erstaktivierung des Implantats erfolgt einige Wochen nach der Operation und besteht aus mehreren Schritten. Zuerst wird getestet, ob alle Elektroden des Implantats funktionsfähig sind. Dabei wird in Abstimmung mit den CI-Träger_innen die Lautstärke-Empfindlichkeit des Systems eingestellt und damit die Anpassung des Sprachprozessors vorgenommen. Erst wenn dieses sogennante Mapping erfolgt ist und die Einstellung auf dem CI-System gespeichert wurde, wird das gesamte Implantat eingeschaltet und ein erster Gesamteindruck des Hörens mit CI stellt sich ein. ${ }^{32}$ Der im Englischen als „switch on“ bezeichnete Moment wird teils auch als ,Umschalten “ (von Nicht-Hören auf Hören) begriffen. ${ }^{33}$ Gerade in populärkulturellen Repräsentationen stellt die Szene der Erstaktivierung ein zentrales Element dar. Auf Video-Sharing-Websites wie Youtube finden sich etliche Amateuraufnahmen, die Kinder und Erwachsene sowie ihre Reaktionen auf das Ereignis „Zum ersten Mal Hören“ zeigen. Es handelt sich größtenteils um Videos, in denen die CI-Träger_innen die Aktivierung positiv aufnehmen, und die zeigen, wie die optisch wie auch akustisch stark emotionalisierende Transformation einer gehörlosen in eine hörende Person hergestellt bzw. vollzogen werden kann. ${ }^{34}$ Die umfangreichen prä- und postoperativen Maßnahmen sowie die mit der Aktivierung gegebenenfalls verbundenen Schwierigkeiten blenden solche Privatfilme in der Regel aus. Im Gegensatz zu diesem Material enthalten die schriftlichen Reflexionen der CI-Träger_innen, die diese in ihren Blogs oder Erfahrungsberichten festhalten, Anhaltspunkte auf welche Weise

31 Für Foucault stellt die Praxis der Übung ein zentrales Element der Subjektkonstitution dar, wobei sie gleichermaßen als Medium eines Disziplinierungs- oder Normierungsprozesses oder als Medium der freien Führung des eigenen Lebens fungieren kann. Im Rahmen der disziplinierenden Übung oder ,subjektivierende[n] Unterwerfung“ sollen - wie unter Punkt 3.3 zu zeigen sein wird „Fähigkeiten der Selbstführung [in diesem Fall des auf die Welt und die Anderen Hörens, B. O.] gewonnen werden, die nach der sozial geteilten Norm für gut befunden werden“". (Vgl. Christoph Menke, Zweierlei Übung. Zum Verhältnis von sozialer Disziplinierung und ästhetischer Existenz, in: Axel Honneth/Martin Saar (Hg.), Michel Foucault. Zwischenbilanz einer Rezeption, Frankfurt a. M. 2003, 283-299, 292).

32 Zum technischen Ablauf der Erstanpassung vgl. Sebastian Hoth, Audiometrie - Die Untersuchung des Gehörs und seine technische Versorgung, in: Rüdiger Kramme (Hg.), Medizintechnik, Berlin u. a. 2011, 217-270, 245.

33 Vgl. Patricia M. Chute/Mary Ellen Nevins, The parents' guide to cochlear implants, Washington, DC 2002, 49.

34 Exemplarisch sei nochmals auf das bereits erwähnte Video von Sarah Churmans Aktivierung verwiesen, das auf Youtube millionenfach abgerufen wurde. 
sich ein anfängliches Hören mit CI gestaltet und wie das Neuroimplantat sich zwischen akustischer Umwelt und den CI-versorgten Personen positioniert. ${ }^{35}$

Einer der beschriebenen Höreindrücke könnte mit dem Begriff des Lärms, wenn nicht gar der Kakophonie gefasst werden. Dabei wird nicht behauptet dass die Gehörlosen oder Schwerhörigen aus einer, absoluten Stille' in die hörende Welt hinübertreten. Vielmehr besteht der kakophonische Höreindruck darin, dass das auditiv Wahrgenommene im Einzelnen noch nicht zugeordnet oder im Verhältnis mit anderen sensorischen, z. B. visuellen Wahrnehmungen eingeordnet werden kann. Vor diesem Hintergrund wird dem Gehörten vielfach ein technischer Charakter zugeschrieben. Peggy Rußat beschreibt den Höreindruck nach der Aktivierung des CI wie folgt:

[...] plötzlich hörte ich ein durcheinander von pfeifen, sirren und brummen. So etwa wie im Dschungel. Wenn man mir was sagte hörte ich nur ein Chaos von Tönen. Etwas irritiert war ich da schon aber nicht enttäuscht. Ich hörte ja was und das war mir viel wert. ${ }^{36}$

Die CI-Trägerin spricht von einem „Durcheinander" und „Chaos“, in dem sich verschiedene Klänge vermischen. Die post-lingual ertaubte Rußat stellt heraus, dass sie das Pfeifen, Sirren oder Brummen in diesem Kontext jedoch nicht als ,Naturklang“ wahrnimmt, sondern eher mit technisch bzw. digital erzeugten Klängen assoziiert. So artikulieren die Ausführungen eine gewisse Befremdung bzw. Unvertrautheit angesichts der akustischen Adressierung durch das CI, die auf den in philosophischen Forschungen zum Hören häufig übersprungenen Unterschied zwischen Hören und Verstehen verweisen. ${ }^{37}$ Es wird folglich eine Relation zwischen CI und akustischer Umwelt hergestellt, während die im Gehirn zu erfolgende Übersetzung der elektrischen Impulse in semantische Einheiten noch nicht etabliert ist. ${ }^{38}$

Ein häufig als Pfeifen wahrgenommenes Geräusch wird von Alexander Görsdorf als mediale Zensurpraxis gedeutet, mit der in Musiktiteln oder im Fernsehen bestimmte Wörter ausgeblendet werden. In seinem Fall ist die Kontrollfunktion jedoch generell auf alles, was gesagt wird, ausgedehnt. Ironisch merkt Görsdorf an:

[...] Fakt ist aber, dass seit das CI an ist, die Leute unglaublich unflätig geworden sind! Nur noch Schimpfwörter, sexuelle Anspielungen und Kraftausdrücke!

35 Vgl. Beate Ochsner/Markus Spöhrer/Robert Stock, Human, Nonhuman, and Beyond. Cochlear Implants in Socio-Technological Environments, in: Beatrice Michaelis/Richard Grusin/Martin Zierold ( $\mathrm{Hg}$.), the re/turn of the nonhuman in the study of culture. concepts - concerns - challenges, Berlin u. a. (im Erscheinen).

36 Peggy Rußat, Mein Leben mit dem Cochlea Implantat, http://cochlearimplanttraegerin.de.tl/ (10.06.2014). Die Blogeinträge enthalten oft Rechtschreib- und Grammatikfehler. Da sich diese häufen wird auf eine Kennzeichnung einzelner Fehler verzichtet. Die Blogeinträge werden so wiedergegeben, wie sie auf den entsprechenden Seiten abrufbar sind (B. O. und R. S.).

37 Vgl. David Espinet, Phänomenologie des Hörens. Eine Untersuchung im Ausgang von Martin Heidegger, Tübingen 2009, 211.

38 Siehe auch eine ähnliche Schilderung bei Görsdorf, der von „Xylofontöne[n] aber gepfiffen, nicht geschlagen“"spricht. Alexander Görsdorf, Das erste Mal. Elektrisches Hören, in: Not quite like Beethoven, 22.12.2009, http://notquitelikebeethoven.wordpress.com/2009/12/22/das-erste-malelektrisches-horen/ (26.06.2014). 
Mein elektrisches Ohr bewahrt mich gnädig davor, es legt einfach einen Piepton über den Blödsinn. Kann ja auch nichts dafür, dass die meisten Leute so durchgängig fluchen, dass ich nur Piepstöne im Rhythmus ihrer Sprache höre. ${ }^{39}$

Enno Park hingegen setzt auf eine onomatopoetische Charakterisierung des Pfeifens ${ }^{40}$ und macht sich ebenfalls daran, den technischen Hintergrund dieses Phänomens zu erklären:

[Der Cyborg hört,] Und zwar einen elektrisch klingenden, an den Rändern etwas diffusen 1200-Hertz-Ton [...]. Diesen Ton höre ich heute seit dem ersten Einschalten des Cochlea-Implantates immer. Jedes Geräusch ist ein kurzes oder langes „Wling”. Sprache ist ein „Wlingwlingwlingewliiingwling” im Rhythmus des Sprechers. Es ist das, was viele als "Xylophon" umschreiben, auch wenn ich nicht finde, dass es so klingt. Dieses ,Wling” wird mich noch bis zu zwei Monate begleiten. Es ist die Grundfrequenz aller elektrischen Impulse, die mein Gehirn leider auch akustisch wahrnimmt, aber bald ignorieren wird. Tatsächlich schälen sich hinter diesem „Wling“ langsam andere Geräusche heraus. Vor allem Geklapper mit Geschirr oder einem Schlüsselbund klingt relativ normal. ${ }^{41}$

Während die oben erwähnten Amateurvideos den Akzent auf die affektive Dimension des ,ersten Hörens ‘ legen, ein Effekt, der sich auch der zeitlichen Konfiguration verdankt (namentlich die simultane Aufzeichnung des Geschehens), verdeutlichen die Blog-Einträge ein breites Spektrum der erst im Nachhinein verschriftlichten Erfahrungen des Einschaltens. Das, was hier aus Sicht der CI-Träger_innen artikuliert wird, markiert das ,switch-on' zudem nicht nur als eine endgültige und scheinbar gelungene Transformation in eine hörende Person, vielmehr wird die Aktivierung des CI als ein erster Schritt einer umfassenden Neuorientierung und sich vollziehenden Subjektivierungsprozess erkennbar.

\subsection{Einstellungsphase}

Die akustische Dimension der Welt gewinnt durch die Aktivierung des CI bzw. seiner Träger_innen (wieder) an Bedeutung. Das heißt nicht, dass die auditive Wahrnehmung vorher nicht gegeben war, aber die Situation des In-der-Welt-Seins ändert sich durch die (Rück-)Kopplung von Mensch und Welt in einem ökotechnologisch

39 Alexander Görsdorf, Die Welt mit CI. Für Kinder und Jugendliche unter 18 Jahren nicht geeignet, in: Not quite like Beethoven, 24.12.2009, http://notquitelikebeethoven.wordpress. com/2009/12/24/ci-fur-kinder-nicht-geeignet/ (26.06.2014).

40 Mit Techniken der Lautmalerei werden hier akustische Phänomene nicht nur nachzuahmen versucht, wie es bei Dichtern der Romantik und anderen der Fall war. Im Gegensatz dazu waren die Wortschöpfungen in den Werken von Dada-Künstlern wie Hugo Ball und Kurt Schwitters eher einer „,nihilistischen Sinnverweigerung“ verpflichtet und ließen nicht unbedingt Schlüsse auf bestimmte akustische Begebenheiten zu. Vgl. Hans Emons, Sprache als Musik, Berlin 2011, 47.

41 Enno Park, Der Cyborg hört, in: die ennomane, 24.05.2011, http://www.ennomane. de/2011/05/24/der-cyborg-hort/ (18.06.2014). 
grundierten Sinnregime. ${ }^{42}$ So verändert sich das Verhältnis der Sinneswahrnehmungen zueinander ebenso wie die bei CI-Träger_innen bis zum Zeitpunkt der Implantation etablierte und anhand der Kombination mehrerer Sinne gekennzeichnete Wahrnehmung der Umwelt. ${ }^{43}$ Das CI produziert zuallererst die Geräusche der Dinge und Menschen, sowohl die der Umwelt wie auch diejenigen, die von den CI-Träger_innen selbst verursacht werden. Nicht der Bach oder das Meer „rauscht“, nicht der Motor „brummt““. ${ }^{44}$ Vielmehr ist es nun die Neuroprothese, die Klänge aufnimmt, prozessiert und dem Hörnerv zuführt, damit dieser die elektrischen Impulse an das Gehirn weiterleitet, das daraus einen Höreindruck generiert. Dabei schaltet sich das CI zwischen CI-Hörende und die akustische Welt, so dass zumindest in der anfänglichen Phase des CI-Hörens das CI nicht verschwindet, sondern die ,Künstlichkeit des Hörens auf unterschiedliche Weise herausstellt.

Die beginnende Immersion in die Welt als ein durch akustische Phänomene gekennzeichnetes Feld wird in den Schilderungen von Görsdorf angedeutet. Nach Aktivierung seines $\mathrm{CI}$ beschreibt er die spielerische Art und Weise, sich die Welt der Töne und Geräusche wieder anzueignen:

Ich klatsche in die Hände, klopfe an Wände und auf Tische, kratze mit den Fingernägeln am Computer, knistere mit der Chips-, der Lebkuchen- und der LidlTüte. Ich stampfe auf den Boden und schleife meine Füße, was beim Gehen durch die Stadt recht komisch aussieht. Ich sage dabei A! und a! und sss! und Pft! vor mich hin, drehe die Dusche an und lasse sie gegen den Duschvorhang pladdern. [...] Leider waren am Ententeich keine Enten, die für mich quaken wollten. Aber - alles macht Geräusche! Und wichtig ist das deswegen, weil ich die (meisten) zwar vorher auch gehört habe, aber jetzt klingen sie wieder spannend, frisch und knusprig statt des ollen, verwaschenen Impressionismus, den ich gewohnt bin. ${ }^{45}$

Der Körper des CI-Trägers wird zum Ausgangspunkt einer Reihe von Geräuschereignissen. Letztere werden sowohl vom Körper selbst (durch Klatschen und sprachliche Äußerungen), als auch im Wechselspiel mit der Umwelt (klopfen, kratzen, schleifen

42 Erich Hörl, Die technologische Bedingung. Zur Einführung, in: ders. (Hg,), Die technologische Bedingung: Beiträge zur Beschreibung der technischen Welt, Berlin 2011, 10.

43 Im Fall von Gehörlosigkeit und Schwerhörigkeit kommt dem Sehen häufig die andere Stellung bei der Wahrnehmung von Welt zu, als dies bei Hörenden der Fall ist. Vgl. Annette Höhne, Eine Welt der Stille. Untersuchungen zur Erfahrungswelt Gehörloser als Ausgangspunkt für eine phänomenologisch-orientierte Gehörlosenpädagogik, München 2005, 89-96.

44, ,[B]ei der Identifikation des Akustischen mit seiner Quelle [ist] zu beachten, dass Geräusche dann weniger als Eigenschaften der sie verursachenden Gegenstände gelten, denn als deren aktivitätsbetonte Äußerung: Etwas hat eine Farbe, aber es macht ein Geräusch." Sybille Krämer, Negative Semiologie der Stimme. Reflexionen über die Stimme als Medium der Sprache, in: Cornelia EppingJäger/Erika Linz (Hg.), Medien/Stimmen, Köln 2003, 65-84, 67 (Hervorh. im Original). Zu einer Übersicht von Verben, mit denen Höreindrücke übersetzt werden, vgl. Joachim-Ernst Berendt, Nada Brahma. Die Welt ist Klang, Reinbek bei Hamburg 1985, 118-120.

45 Alexander Görsdorf, Die Lehren des Musiklehrers, in: Not quite like Beethoven, 24. 12. 2009, http://notquitelikebeethoven.wordpress.com/2009/12/23/die-lehren-des-musiklehrers/

(26.06. 2014). Der „olle verwaschene Impressionismus“ bezieht sich auf das Hören mit Hörgeräten. 
an Gegenständen oder dem Fußboden) erzeugt und sind in unterschiedlichen Umgebungen (Innenräume, Supermarkt, Straße) verortet. Doch scheinen diese „Geräusche“ nicht nur „spannend“ und „frisch“, sondern auch „elektrisch“, wie Görsdorf in einem Blogeintrag wenig später vermerkt: „Ansonsten klingt durch Gras laufen heute, als wären es Metallstäbchen, Blätter klingen wie Metallfolien. Und auch der Springbrunnen klingt als prallten die Tropfen auf ein Blechdach und nicht die Wasseroberfläche. “46 Dass die Klangerzeugung dem CI geschuldet ist, dessen Signale das Gehirn der CI-Träger_innen erst erlernen wird, zeigt ebenfalls die Darstellung Michael Chorosts über seine anfängliche Re-Situierung. Er fühle sich wie ein Kind, als er zufällig den Klang wiederentdeckt, den Blätter machen:

There are large fibrous magnolia leaves scattered on the ground [...]. Absentmindedly, I kick one. It tinkles. As it skitters along the ground, it makes a tinny, tinfoily noise as its edges scrape along the concrete. [...] I find this deeply interesting. It brings out the six-year-old in me. As I make my way around the building, I go out of my way to kick leaves, sometimes booting a particularly noisy leaf ahead of me three or four times to get maximum entertainment value out of it. Each time, the leaf goes clitter clitter clitter clitter. ${ }^{47}$

Anhand der Beschreibung wird ersichtlich, wie Gegenstände und Geräusche voneinander getrennt und die Geräusche als Äußerung der Gegenstände zudem durch das CI geformt werden. Besonders deutlich macht dies die onomatopoetische und sich wiederholende Artikulation des Geräusches der Blätter durch die Fußstöße. Dabei verwendet Chorost nicht das Verb ,rustle (rascheln)“, sondern das Wort „,clitter“, das ein Reibungsgeräusch, Geklirr oder Geklapper bezeichnet. ${ }^{48}$ Sowohl Beschreibung als auch Wortwahl verweisen aber nicht nur auf die spezifische Qualität des CI-Hörens, sondern auch auf eine neue Art Kartographierung der akustischen und damit auch der (laut)sprachlichen Umwelt durch und mit dem CI. Ähnlich wie Görsdorf und Park empfindet auch Chorost das CI-Hören als ,,interessant“. Der durch das Implantat verfremdete Klang wird geradezu als exotisch stilisiert. Es handelt sich um eine Wahrnehmungsweise, die scheinbar CI-Träger_innen vorbehalten ist. Diese können sich somit nicht nur von Nicht-, sondern in gleichem Maße von Normal-Hörenden abgrenzen. Ihre Anziehungskraft beziehen solche von der Norm abweichenden Wahrnehmungen aber sicher auch daher, dass es sich dabei um eine zeitlich begrenzte Form des Höreindrucks handelt, der aufgrund der schrittweisen Etablierung eines spezifischen auditiven Erwartungshorizonts bzw. durch die zunehmende Gewöhnung und Bindung an die sprachlich-semantische Ordnung verwischt wird. ${ }^{49}$

46 Alexander Görsdorf, Die Welt mit CI. Für Kinder und Jugendliche unter 18 Jahren nicht geeignet, in: Not quite like Beethoven, 24.12.2009, http://notquitelikebeethoven.wordpress. com/2009/12/24/ci-fur-kinder-nicht-geeignet/ (26.06.2014).

47 Michael Chorost, Rebuilt. How becoming part computer made me more human, London 2006, 54, Hervorhebung im Original.

48 Vgl. Clitter. Merriam-Webster, http://www.merriam-webster.com/dictionary/clitter (02.07.2014).

$49 \mathrm{Vgl}$. Görsdorf, Das erste Mal. 
Das Zurechtfinden in der Welt der Geräusche, die durch das CI initiiert wird, schließt auch Umgebungsgeräusche ein. Diese werden nicht von den Personen selbst erzeugt und werden von Hörenden in der Regel als Bestandteil eines Grundklangs bestimmter räumlicher Verhältnisse (zum Beispiel Wohnung oder Stadt) durch Gewöhnung überhört. ${ }^{50}$ Peggy Rußat schreibt in ihrem Blog, wie sie nach der Erstaktivierung auf dem Weg nach Hause von einer „Flut von Tönen“ überwältigt wurde. In den eigenen vier Wänden angekommen, legte sie eine Hörpause ein:

Als ich im stillen Wohnzimmer saß, hörte ich nach ein paar Minuten Stille ganz leise ein klacken. [...] Als mein Mann wieder ins Wohnzimmer kam fragte ich ihn, ob die Uhr (fand erst einmal nichts anderes, was klacken könnte) über dem Fernseher eigentlich tickt. Er schaute mich ungläubig an und schüttelte dann mit dem Kopf. Ich hörte das klacken aber immer noch. Ich nahm daraufhin die Uhr ab und hielt sie mir ans CI. Natürlich klackte diese Uhr. Mein Mann hatte das Ticken nach all den Jahren nicht mehr registriert. ${ }^{51}$

Die in diesem Abschnitt erörterte Re-Situierung der CI-Träger_innen scheint sich anfänglich, d. h. in den ersten Tagen nach der Aktivierung, eher spontan oder sogar spielerisch zu ereignen, indem sie auf akustische Phänomene durchaus unterschiedlich reagieren. Dies, so zeigen die Selbstbeschreibungen der CI-Träger_innen in den Blogs ebenfalls, wandelt sich jedoch in zunehmenden Maße, wenn es um das (Wieder-) Erlernen bestimmter Fähigkeiten geht, wie z. B. dem Sprach- oder Musikverstehen.

\section{3 Übung}

Der Philosoph Christoph Menke definiert im Anschluss an Foucault zwei spezifische Formen von Übung als Praktiken der Selbsttechnologie. Dabei unterscheidet er zwischen der Einübung bestimmter Fertigkeiten, welche die Beziehung zu sich selbst regulieren und mit Machtgewinn verbunden sind, und körperlichen Normierungstechniken, die im Kontext einer lebenslangen Verbesserung und Disziplinierung von Fähigkeiten zu begreifen sind. ${ }^{52}$ Während Menke hinsichtlich der ersten Form von Übung, die er als Ästhetik der Existenz bezeichnet, von einem Zuwachs an Autonomie des Subjektes ausgeht, so gerät die Selbstbestimmung im oben beschriebenen disziplinierenden Modus der zweiten Übung zu einer Selbstunterwerfung und dem Erwerb von Handlungsfähigkeit (hier: Hörfähigkeit) mit dem Ziel, ,normal ‘ bzw. der sozialen Norm entsprechend zu funktionieren. Der Prozess der Norm(alis)ierung des Hörens und die Ergebnisse, die beim Sprachverstehen und Musikhören erreicht

50 Vgl. Bernhard Waldenfels, Stimme und Echo, Blick und Spiegel, in: ders./Giuliani Regula (Hg.), Das leibliche Selbst. Vorlesungen zur Phänomenologie des Leibes, Frankfurt a. M. 2000, 379387, 381; Siegfried Saerberg, Konstitution des Raumes im blinden und sehenden Wahrnehmungsstil, „Geradeaus ist einfach immer geradeaus.“ Eine lebensweltliche Ethnographie blinder Raumorientierung, Konstanz 2006, 87-112, 95.

51 Peggy Rußat, Mein Leben mit dem Cochlea Implantat, http://cochlearimplanttraegerin.de.tl/ (10.06. 2014). Für eine ähnliche Beschreibung vgl. Graham M., Ticka ticka ticka ticka ticka, in: Gray's Deaf Blog, 13.06.2010, http://graysdeafblog.wordpress.com/2010/06/13/ticka-ticka-tickaticka-ticka/ (02.07.2014).

52 Vgl. Menke, Zweierlei Übung. 
werden können, sind dabei stark vom persönlichen Einsatz der CI-Träger_innen abhängig. Das Praktizieren von Hör-Übungen wird so selbst zu einem Element eines Regimes von Selbsttechniken. Die Übung stellt mithin eine Arbeit an sich selbst dar und ist Teil eines disziplinierenden Subjektivierungs- und Transformationsprozesses, der letztlich ein CI-hörendes Subjekt (bzw. ein hörendes Subjekt, das sich eben doch erneut durch das CI unterscheidet) hervorbringen soll. Der Erfahrungsbericht von Anja, CI-Trägerin, auf der Homepage des Geräteherstellers Med-El bringt dies deutlich zum Ausdruck:

Success with a CI is really dependent on oneself, especially how committed one is to practicing listening after the operation. This also gave me confidence, because I am a fighter and I knew it just had to work for me. The possibility of better hearing was solidly in my mind and wouldn't let go. Deep inside of me I knew that I already decided to go this direction and nothing would stop me. ${ }^{53}$

CI-Herstellerfirmen nutzen solche Erfahrungsberichte - auch in Buchform - zur Verbreitung von Erfolgsgeschichten. Über Misserfolge ist hier selten zu lesen. Im Fall des zitierten Beispiels kommt noch ein weiterer Aspekt ins Spiel. Es geht hier darum, die Erwartungshaltungen von potenziellen CI-Implantierten zu begrenzen. Dem Implantat-System wird damit tendenziell abgesprochen, von selbst und ohne die aktive Beteiligung der Implantierten zu funktionieren. Vielmehr erzeugt Anjas Bericht den Eindruck, dass das Implantat etwas ist, dessen Einsatz effektiv von den Fähigkeiten und dem Willen der Nutzer_innen abhängt. Das Versprechen, (wieder) hören zu können, erfordert die aktive Mitarbeit des Patienten, dem nach der Implantation die Verantwortung für Erfolg oder Misserfolg der unter Umständen langwierigen Behandlung übertragen wird. Auf diese Weise werden nach der Implantation nicht in erster Linie Hersteller-Firmen, Audiologen oder Ärzte für den erfolgreichen Umgang mit dem CI verantwortlich gemacht, sondern die Implantierten selbst. Im gleichen Zug kann ein mögliches Scheitern als selbsttechnologisches Versagen oder als Verweigerung und mithin als (Selbst-)Ausschluss aus der Gemeinschaft gewertet werden.

Aus anderen Beschreibungen von CI-Träger_innen geht hervor, dass mit HörÜbungen bestimmte Praktiken und Bedingungen verbunden sind, die u. a. die zeitliche Strukturierung des Tagesablaufs betreffen und zu vordergründig nicht intendierten Nebeneffekten führen können:

Since going bionic, I've started reading books again in a big way. [...] I'm reading one chapter from each of four audiobooks each day. A chapter from an audiobook can be between five and fifteen minutes, so that adds up to around an hour's reading time a day in total. [...] I'd long lost the ability to concentrate on one single task at a time. Now, with these audiobooks, I find my mind becoming an oasis of calm. One activity, one purpose. ${ }^{54}$

53 Anja, Anja's Diary, in: Med-El. Testimonials, http://www.medel.com/us/support-testimonialsanja/ (16.06.2014).

54 William Mager, Billy 2.0. Learning to read again, in: The Limping Chicken, 02. 04. 2013, http:// 
Die Hör-Übungen sind zudem mit einer bestimmten körperlichen Haltung und Situierung verbunden. Die CI-Träger_innen begeben sich z. B. in eine akustische Isolation, indem sie sich direkt mit Musikabspielgeräten verbinden, so dass Umgebungsgeräusche ausgestellt werden. Diese Zumutung wird positiv umgedeutet, denn sie wird als Voraussetzung einer zukünftigen besseren Hörfähigkeit verstanden. Mager schreibt dazu:

When I plug myself directly into the headphone socket, all the outside noises go away and it's just me, an iPad, and someone telling me a story that I've heard before, but I don't mind hearing again and again. [...] This is all part of my long term training montage - but is it working? I think it is. [...] Behind the scenes, my brain is pedalling furiously on an exercise bike, a couple of earbuds jammed into its ears. ${ }^{55}$

Die Übung in Isolation wird wiederholt, um das Sprachverstehen über das CI zu verbessern und um damit später in Alltagssituationen über ein höheres Maß an Kommunikationsfähigkeit verfügen zu können. Diese Praxis des Lernens im Rückzug geschieht möglicherweise auch, weil sich erwachsene Spätertaubte bzw. nunmehr CI-Träger_innen nicht in ein Lautsprachtraining - im Sinne Jürgen Trabants eine Konfiguration der Hörigkeit ${ }^{56}$ - begeben möchten, wie Görsdorf dies beschreibt:

Ich habe kein Programm auferlegt bekommen. Bin da ganz frei. Mache und höre, was ich will. Oder lasse es. Versuche aber, möglichst viel zu üben. [...] Mit Personen hab ich nie explizit geübt. Ich hätte mich da auch nicht wohlgefühlt, wenn Leute vor mir sitzen und ständig ein Wort wiederholen. Mmmmandel. Mmmaaaaaandel. Mmmannndddell1111111. ${ }^{57}$

Andere, die bereits seit ihrer Kindheit mit logopädischem Unterricht konfrontiert sind, berichten das Gegenteil. So schreibt Fiona Bollag über das Sprachtraining mit ihrer Therapeutin:

Frau Schmidt-Giovannini half mir, sie übte mit mir. Ich liebte sie, bei aller notwendigen Strenge hatte sie Geduld und unglaubliche Erfahrung. Die Stunden waren sehr intensiv und anstrengend. [...] Neben der Schule und den anderen Fächern musste ich mich voll auf den Sprachunterricht konzentrieren, aber ich

limpingchicken.com/2013/04/02/william-mager-billy-2-0-learning-to-read-again/ (17.06.2014). Vgl. dazu auch die Erfahrungen des Users Kim unter: Post Activation Day, in: Face me, 18. 12. 2011, http://faceme.wordpress.com/2011/12/18/post-activation-day/ (17.06.2014).

55 William Mager, Billy 2.0. Learning to read again.

56 Vgl. Jürgen Trabant, Sprache und Hörigkeit des Menschen, in: Dietmar Kamper/Christoph Wulf (Hg.), Anthropologie nach dem Tode des Menschen: Vervollkommnung und Unverbesserlichkeit, Frankfurt a. M. 1994, 279-304, 282-289.

57 Alexander Görsdorf, Jetzt mal im Ernst: Hören Lernen mit dem Cochlea Implantat, in: Not quite like Beethoven, 11.03.2010, https://notquitelikebeethoven.wordpress.com/2010/03/11/horenlernen-cochlea-implantat/ (26.06.2014). 
wollte es ja lernen, und wie ich es wollte! Lippen ablesen war nun ganz verboten, und Frau Schmid-Giovannini wiederholte die Sätze so lange, bis ich sie mit geschlossenen Augen verstand. Sie erzählte Geschichten, die ich nur über das Ohr aufnehmen durfte, und ich musste sie nacherzählen..$^{58}$

Auf diese Weise werden einerseits unter Umständen lange Jahre einer anderen Hörerfahrung überschrieben, das CI-Hören wie auch die CI-Hörenden durch die Regularien ihrer (Selbst-)Verortung in der hörenden Welt relativiert und ins System der Hörnorm integriert. Andererseits aber bleibt die Differenz zwischen CI-Hören und Normalhören erhalten, wenn nicht durch die Sichtbarkeit des Gerätes selbst, so doch vor allem durch die stets betonte Orientierung am vermeintlich natürlichen Hören, in Bezug auf welches das CI-Hören automatisch als abweichend oder erklärungswürdig markiert wird. Dabei wird jedoch nicht nur das ,andere' Hören, sondern immer auch das Normalhören als eine kulturelle Praxis unter anderen sichtbar. Mit Joseph Vogl kann in Bezug auf diese sich beständig wandelnde Konstellation von einer „Denaturierung“ des (Normal-)Hörens gesprochen werden, die die Geschichte des Hörens als solches als einer medialen Praxis eröffnet. ${ }^{59}$ Mit dem Erlernen des CI-Hörens ist folglich die Inkorporierung eines technischen Device verknüpft, welches die akustische Wahrnehmung(sfähigkeit) nach und nach an der Hörwelt bzw. der Welt der Hörenden nach- beziehungsweise als eigene Praxis ausbildet. Insofern können die Hörübungen als ein Prozess des Medium-Werdens im Sinne Vogls beschrieben werden, in dessen Verlauf das CI zum Mediator zwischen Träger_in und akustischer Welt wird, ihr mögliches Rauschen zunächst zum anfänglichen Störgeräusch erklärt und dieses dann wiederum verschwinden lässt.$^{60}$ So ist das CI gerade nicht als zeitresistentes technisches Objekt zu verstehen, vielmehr wird es - und dies zeigt sich an und in den Hörpraktiken - ebenso wie sein/e Träger_in in Bezug auf das, was ,normales' Hören bedeutet, eingestellt und somit im Gebrauch hergestellt. ${ }^{61}$ Auf diese Weise wird ein neues oder anderes Verständnis des Verhältnisses zwischen Umwelt, Ohr, Hören (und zugleich Nicht-Hören) und Gehörtem (wie zugleich Nicht-Gehörtem) erzeugt. Es handelt sich um einen Prozess, in dem sich die Transformation des CI vom reinen Instrument zum Medium ereignet. ${ }^{62}$ In den Dispositiven von Implantation, Aktivierung und Einstellung wird das Hören durch das CI um die Reflexion des (Normal-)Hörens und seine Gesetzmäßigkeiten ergänzt bzw. ersetzt und lässt - nach einer gewissen Zeit - auch nichts (mehr) hören, was nicht auch das Normalhören hören ließe.

58 Fiona Bollag/Peter Hummel/Angela Kuepper, Das Mädchen, das aus der Stille kam, Bergisch Gladbach 2006, $166 \mathrm{f}$.

59 Joseph Vogl, Medien-Werden. Galileis Fernrohr, in: Archiv für Mediengeschichte 1 (2001), $115-123,115$.

60 Vgl. Alexander Görsdorf, Wie hören Menschen mit Cochlea Implantat? in: Not quite like Beethoven, 08. 01.2010, http://notquitelikebeethoven.wordpress.com/2010/01/08/wie-horen-menschenmit-cochlea-implantat/ (26.06.2014).

61 Vgl. Joseph Vogl, Taming time. Media of Financialization, in: Grey Room 56 (2012), 72-83.

62 Vgl. Vogl, Medien-Wenden, 121. 


\section{Hörszene und mediale Subjektivierungspraxis}

Die Analyse der Selbstbeschreibungen von CI-Träger_innen zeigt, dass das CI-Hören als Übersetzungsprozess ${ }^{63}$ und individuelle Subjektivierungspraxis konzeptualisiert werden kann, die im Gegensatz zum eingangs erwähnten Einstellungsprozedere der Audiologen steht. Des Weiteren wird anhand der autobiographischen Schilderungen die Relevanz der Körperlichkeit des Hörens erkennbar, die in philosophischen, ${ }^{64}$ aber auch medizinischen ${ }^{65}$ Reflexionen zugunsten des Aspekts des sinnhaften Verstehens oft vernachlässigt wird. In dieser Perspektive erscheint das CI als Mediator, der zwischen hörgeschädigten bzw. gehörlosen Personen und der Welt der Geräusche und Sprache positioniert ist, als Medium dann aber nur in seinem Gebrauch sowie in den Praktiken der (Selbst-)Beschreibungen erkennbar gemacht werden kann. ${ }^{66}$

Auf einer weiteren Ebene realisiert das Implantat-System - quasi im Sinne eines Vermittlungsprozesses, an dem Medizin, Audiologie und Gerätehersteller beteiligt sind - eine Form der Interpellation ${ }^{67}$ von Spätertaubten und Gehörlosen im Sinne Louis Althussers und ist auf diese Weise intrinsisch am Subjektivierungsprozess der solchermaßen Angerufenen beteiligt. In diese Konfiguration sind eine Reihe von Diskursen eingeschrieben, die von der Medikalisierung von Gehörlosigkeit über die Normalisierung von Nicht-Hörenden bis zur Bildung oder Auflösung bestimmter Gemeinschaften, z. B. der Gehörlosen-Community oder CI-Hörenden, reichen. ${ }^{68}$ Da sich hier jedoch diverse Zuschreibungen in Bezug auf das, was das CI ist, was es nicht ist oder was es sein soll, auf mehrfacher Ebene kreuzen und das CI zudem selbst als nichtmenschlicher Akteur auf das Geschehen einwirkt, ist es notwendig, das Konzept der Interpellation im Sinne Althussers in einer dezentrierten und entideologisierten Weise zu erweitern. Denn die Instanzen und Akteure, die am Prozess des Anspre-

63 Vgl. A. Hahne, L. Bruns, D. Mürbe, Semantische Verarbeitung komplexer Musikstücke nach Cochlea-Implantation. Elektrophysiologische Evidenz, Vortrag auf der Jahrestagung der Deutschen Gesellschaft für Audiologie, Oldenburg, 12.-15 032 014, www.uzh.ch/orl/dga2014/programm/wissprog/Hahne.pdf (10.07.2014). S. a. die Ausführungen von Davis, der als Kind gehörloser Eltern (CODA) aufwuchs. In seinen Memoiren beschreibt Davis, wie er in seiner Kindheit ein großes Vokabular entwickelte, um seinen Eltern seine Welt der Geräusche zu erklären. Vgl. Lennard J. Davis, My sense of silence. Memoirs of a childhood with deafness, Urbana 2000, 2.

64 Vgl. dazu David Espinet, Das Lied der Erde. Zu Heideggers Phänomenologie des Hörens als Ontologie der Halbdinge, in: Steffen Kluck/Stefan Volke (Hg.), Näher dran? Zur Phänomenologie des Wahrnehmens, Freiburg u. a. 2012, 273-300.

65 Vgl. u. a. Eisen, Djourno, u. a. 503.

66 Vgl. Vogl, Medien-Werden.

67 Vgl. Louis Althusser, Ideologie und ideologische Staatsapparate (Anmerkungen für eine Untersuchung), in: ders., Ideologie und ideologische Staatsapparate. Aufsätze zur marxistischen Theorie, Hamburg 1977, 108-153, 138-145. Althusser beschreibt sein Konzept anhand einer Schlüsselszene, in der ein beliebiger Spaziergänger von einem Polizisten mit , He, Sie da' adressiert wird. Die Subjektwerdung des Spaziergängers wird hierbei im doppelten Moment des Ansprechens selbst sowie des sich als Angesprochenen erkennenden bzw. identifizierenden Subjektes verortet. Die Anrufung wird insofern als ein unumgehbarer Prozess verstanden, im Rahmen derer sich die Subjektwerdung als Unterwerfung von Individuen in einem Machtgefüge ereignet.

$68 \mathrm{Vgl}$. Hayagreeva Rao, Market rebels. How activists make or break radical innovations, Princeton, NJ u. a. 2009, 9; Michele Friedner, Biopower, Biosociality, and Community Formation. How Biopower Is Constitutive of the Deaf Community, in: Sign Language Studies 10 (2010) H. 3, $336-347$. 
chens im Umfeld des CIs beteiligt sind, stellen sich als vielfältig dar und scheinen vor allem nicht immer in dem Maße gefügig zu sein, wie Althusser dies mit dem „Ruf des Polizisten“" nahelegt. ${ }^{69}$ Nicht alle Spätertaubten und Gehörlosen reagieren gleichermaßen enthusiastisch auf den Appell, sich der Welt der Hörenden zuzuwenden, sondern begreifen das CI eher als eine Art „Zwangsbeglückung“, der sie vehement widersprechen. ${ }^{70}$ Während also bei Althusser die Möglichkeit des Nicht-Hörens ausgeschlossen scheint, muss im Handlungszusammenhang CI auch das nicht semantische CI-Hören sowie auch das durch Ausschalten des CIs (re-)initiierte Nicht-Hören bedacht werden. ${ }^{71}$ Solche Praktiken wären im Anschluss an Judith Butler als subversiv zu verstehen, da sie sich gegen die Aufforderung zum Hören wenden bzw. diese anders deuten. ${ }^{72}$ Des Weiteren ist das Hörereignis nicht als einmalig zu denken. Die Praktiken des CI-Hörens sind vielmehr auf eine ständige, nicht identische Wiederholung angelegt, in deren Wiederholungen sich Widerständigkeiten artikulieren. ${ }^{73}$ In dem Maße, wie sich räumliche Gegebenheiten, Distanzen und Rede-Lautstärken sowie auch Personenkonstellationen ändern, verändern sich die Möglichkeitsbedingungen des CI-Hörens und der damit verknüpften Subjektivierungsprozesse. Außerdem sind diese - wie gezeigt wurde - nicht nur situativ bedingt, vielmehr durchlaufen sie bestimmte Phasen wie Erstaktivierung, Einstellung oder Umstellung bei Systemwechsel. In Anlehnung an das Konzept der Schreibszene, wie es u. a. von Martin Stingelin entworfen wurde, kann die mit dem CI verbundene mediale Praxis als eine Hörszene begriffen werden. ${ }^{74}$ Die von Stingelin bestimmten Charakteristika der Körperlichkeit, Instrumentalität und Sprachlichkeit können auf das Hören übertragen werden. ${ }^{75}$ Alle drei Dimensionen stellen sich als Quelle von Widerständen dar, die im Schreiben bzw. Hören selbst überwunden werden (müssen). Stingelin zufolge ist dies als politischer Akt zu verstehen, in dem die (vorauszusetzenden) Bedingungen des Schreibens im

69 Vgl. Ulrich Bröckling, Der Ruf des Polizisten, in: Reiner Keller/Werner Schneider/Willy Viehöver (Hg.), Diskurs - Macht - Subjekt, Wiesbaden 2012, 131-144.

70 Vgl. Ewald Feyerer, Offene Fragen und Dilemmata bei der Umsetzung der UN-Konvention, in: Zeitschrift für Inklusion, (2): http://www.inklusion-online.net/index.php/inklusion-online/article/ view/89/89 (22.07.2014); Beate Ochsner, Teilhabeprozesse oder: Das Versprechen des CochleaImplantats, in: AugenBlick. Konstanzer Hefte zur Medienwissenschaft (2013) H. 58. Themenheft: Objekte medialer Teilhabe, 112-123.

71 Vgl. Linda M. Watson/Susan Gregory, Non-use of cochlear implants in children: Child and parent perspectives, in: Deafness and Education International 7 (2005) H. 1, 43-58.

72 Zur Kritik Althussers Interpellationsmodell vgl. Judith Butler, The psychic life of power: Theories in subjection, Stanford 1997, 106-131.

73 Vgl. Judith Butler, Körper von Gewicht. Die diskursiven Grenzen des Geschlechts, Frankfurt a. M. 1997, 173-175.

74 Der Begriff der Hörszene kann auf Herder zurückgeführt werden: Herder begreift den Menschen als denkendes ,sensorium commune“ (296), das die verschiedenartigen Sinneseindrücke zu einem Ganzen zu verbindet. Das Gehör ist dabei „,der Mittlere seiner Sinne, die eigentliche Tür zur Seele, und das Verbindungsband der übrigen Sinne“ (299). So privilegiert er das Ohr und bezeichnet es als „Tür zur Seele“ (ebd.), gleichwohl entsteht dieses Hören aus dem Hören auf die Welt, d.h. eine kommunikativ-pragmatische Dimension des Hörens auf den anderen ist hier nicht der Sprachursprung; dieser verdankt sich der semantischen Sphäre. Zitate entnommen aus: Johann Gottfried Herder, Über den Ursprung der Sprache, München 1987.

75 Vgl. Martin Stingelin, ,Schreiben. ' Einleitung, in: Martin Stingelin/Davide Giuriato/Sandro Zanetti (Hg.), „Mir ekelt vor diesem tintenklecksenden Säkulum.“ Schreibszenen im Zeitalter der Manuskripte, München 2004, 7-21. 
Schreiben selbst wiederholt und reflektiert werden. Für den Fall des CI-Hörens hat sich gezeigt, dass die Bedingungen des Hörens im Akt des Hören(auf)schreibens vollzogen und reflektierbar werden. Dies betrifft insbesondere das mediale Format des Blogs, in dem Schreib- und Hörszene zusammengeführt werden. Durch die Analyse der automedialen Zeugnisse wird deutlich, dass dort, wo das Hören aufgehalten wird oder auf Widerstände stößt, die Rahmung, die seine alltägliche Praxis strukturiert, erst sichtbar gemacht wird. 